\title{
NÚMERO DE CASOS DE LEPRA EN BRASIL DE 2010 A 2015
}

\section{ARTÍ́CULO ORIGINAL}

MONTEIRO, Lis Mayra Dantas¹, FACCO, Lucas², FECURY, Amanda Alves³ ARAÚJO, Maria Helena Mendonça de ${ }^{4}$, OLIVEIRA, Euzébio de ${ }^{5}$, DENDASCK, Carla Viana ${ }^{6}$, SOUZA, Keulle Oliveira da7 ${ }^{7}$ DIAS, Claudio Alberto Gellis de Mattos ${ }^{8}$

MONTEIRO, Lis Mayra Dantas. Et al. Número de casos de lepra en Brasil de 2010 a 2015. Revista Científica Multidisciplinar Núcleo do Conhecimento. Año 05, Ed. 11, Vol. 25, págs. 62-70. Noviembre de 2020. ISSN: 2448-0959, Enlace de acceso: https://www.nucleodoconhecimento.com.br/salud/hanseniase-in-brasil, 10.32749/nucleodoconhecimento.com.br/salud/hanseniase-in-brasil

\section{RESUMEN}

La lepra se considera una enfermedad infecciosa causada por la bacteria Mycobacterium leprae también conocida como Bacillus de Hansen. Además de ser una enfermedad con evolución gradual, la lepra depende de la relación entre el parásito y el huésped. El objetivo de este estudio fue mostrar el número de casos de lepra en Brasil entre 2010 y 2015 en Brasil. La investigación se llevó a cabo en DATASUS (http://datasus.saude.gov.br/). Con el avance de las políticas públicas, el número de casos de la enfermedad en Brasil disminuyó entre 2010 y 2015. Tiene

\footnotetext{
${ }^{1}$ Técnica Minera, como resultado del Instituto Federal de Amapá (IFAP).

2 Estudiante del Curso de Medicina de la Universidad Federal de Amapá (UNIFAP).

${ }^{3}$ Biomédica, Doctora en Enfermedades Tropicales, Profesora e investigadora del Curso Médico de la Universidad Federal de Amapá (UNIFAP).

${ }^{4}$ Médico, Profesor e investigador del Curso Médico de la Universidad Federal de Amapá (UNIFAP).

${ }^{5}$ Biólogo, Doctor en Enfermedades tópicas, Profesor e investigador del Curso de Educación Física de la Universidad Federal de Pará (UFPA).

${ }^{6}$ Teólogo, Doctor en Psicoanálisis, investigador del Centro de Investigación y Estudios Avanzados - CEPA.

${ }^{7}$ Sociólogo, Máster en Estudios Antrópicos en la Amazonía, Miembro del Grupo de Investigación "Laboratorio de Educación, Medio Ambiente y Salud" (LEMAS/UFPA).

${ }^{8}$ Biólogo, Doctor en Teoría e Investigación del Comportamiento, Profesor e investigador del Programa de Posgrado en Educación Profesional y Tecnológica (PROFEPT), Instituto Federal de Amapá (IFAP).
}

$\mathrm{RC}: 68072$

Disponible: https://www.nucleodoconhecimento.com.br/salud/hanseniase-in-brasil 
una mayor prevalencia en los hombres y la tasa más alta de pacientes es en grupos de edad más grandes, es decir, en adultos. El período de incubación de la enfermedad (que puede variar de 2 a 7 años). En vista de esto, se pueden desencadenar varios problemas a nivel individual, como las afectaciones físicas debilitantes y psicológicas. Con el fin de reducir el número de casos de la enfermedad en el país y poder hacer el diagnóstico lo antes posible, se deben analizar los hallazgos clínicos y las pruebas disponibles para esta detección.

Palabras clave: Epidemiología, lepra, Mycobacterium leprae.

\section{INTRODUCCIÓN}

La lepra se considera una enfermedad infecciosa, ya que necesita contacto directo con el bacilo para transmitir entre las personas. Es causada por la bacteria Mycobacterium leprae también conocido como Bacillus de Hansen. Esta enfermedad sólo se detecta, en promedio, entre 2 y 7 años de incubación y los síntomas suelen aparecer de 11 a 16 días. En esta fase sintomática, la transmisión interpersonal es posible, y por lo general sólo una pequeña porción cae enferma (BRASIL, 2002; OLIVEIRA et al., 2016).

La lepra se puede clasificar en dos formas: lepra paucibacillaria o lepra multibacillaria. En la variación Paucibacilar, el paciente tiene resistencia a los bacilos, presentando hasta cinco lesiones cutáneas. Los bacilos son superados en número en el cuerpo y no son suficientes para la transmisión a otras personas. En este caso existe la posibilidad de curar espontáneamente. La lepra multibacillar es parte del llamado grupo transmisor, con seis o más lesiones cutáneas. Los enfermos no presentan una defensa contra la bacteria, eliminándolas por las vías respiratorias (BRASIL, 2002; BRASIL, 2016). El contagio generalmente ocurre a través del contacto directo entre una persona susceptible y un paciente no tratado, generalmente algún miembro de la familia (BRASIL, 2015).

$\mathrm{RC}: 68072$

Disponible: https://www.nucleodoconhecimento.com.br/salud/hanseniase-in-brasil 
Además de ser una enfermedad con evolución gradual, la lepra depende de la relación entre el parásito y el huésped. Cuando el huésped tiene un sistema de defensa eficiente, tiende a no manifestar la enfermedad. Por diversas razones, como la vulnerabilidad socioeconómica y la convivencia con muchas personas en el mismo entorno, puede llegar a individuos de diversas edades y ambos sexos (BRASIL, 2002).

Los síntomas pueden aparecer en la piel o el sistema nervioso. En la piel se caracterizan generalmente por lesiones con pérdida o aumento de la sensibilidad. Pueden aparecer manchas de pigmento, placas, infiltraciones, tubérculos $\mathrm{y} / \mathrm{o}$ nódulos. En el sistema nervioso los síntomas pueden aparecer como discapacidades físicas y, dependiendo del grado de la enfermedad, deformidades en las extremidades del cuerpo (BRASIL, 2002; FREITAS, 2015).

El tratamiento de la lepra se realiza con la combinación de varios medicamentos, llamados poliquioterapia o MDT. Para los pacientes con la forma Paucibacillar, se utilizan dos tipos de antibióticos. Para aquellos con forma multibacilar, se recomiendan tres tipos de antibióticos (BRASIL, 2002; FREITAS, 2015).

Según el Ministerio de Salud, el número de nuevos casos en Brasil durante los años encuestados fue de 193.021 pacientes. (BRASIL, 2017).

\section{OBJETIVO}

Mostrar el número de casos de lepra en Brasil entre 2010 y 2015 en Brasil.

\section{MÉTODO}

La investigación se llevó a cabo en DATASUS (http://datasus.saude.gov.br/), y los datos de la lepra se recopilaron de los siguientes pasos: en la pestaña "Acceso a la información", se seleccionó "Información de salud (TABNET)" e inmediatamente

RC: 68072

Disponible: https://www.nucleodoconhecimento.com.br/salud/hanseniase-in-brasil 
después de hacer clic en "Epidemiología y morbilidad". En la siguiente página, haciendo clic en "Casos de lepra - Desde 2001 (SINAN)", se eligió la opción "Lepra desde 2001", y en ámbito geográfico optamos por "Brasil por región y unidad de la Federación". Los datos se recopilaron de 2010 a 2015, eligiendo: en línea las opciones "Año Diagnóstico", "Región", "Género", "Grupo de Edad", "Modo de detección", "Evaluación diagnóstica" y "Lesion de la piel". En el campo de columna se utilizó la opción "No activo" y en contenido la frase "Pacientes para la atención" para las opciones de línea anteriores. Después de la selección, estos datos se compilaron en la herramienta De Excel, el componente de Office de Microsoft Corporation. Investigación bibliográfica realizada en el laboratorio del Instituto Federal de Educación, Ciencia y Tecnología de Amapá, Campus Macapá, ubicado en: Rodovia BR 210 KM 3, s/n - Bairro Brasil Brasil. CEP: 68.909-398, Macapá, Amapá, Brasil.

\section{RESULTADOS}

La Figura 1 muestra el número de casos de lepra en Brasil entre 2010 y 2015 . Se observa que en los últimos años se ha observado una disminución en el número de casos, con un pequeño aumento en el número de 2014 en comparación con 2013.

RC: 68072

Disponible: https://www.nucleodoconhecimento.com.br/salud/hanseniase-in-brasil 
Figura 1 Número de casos de lepra en Brasil entre 2010 y 2015.

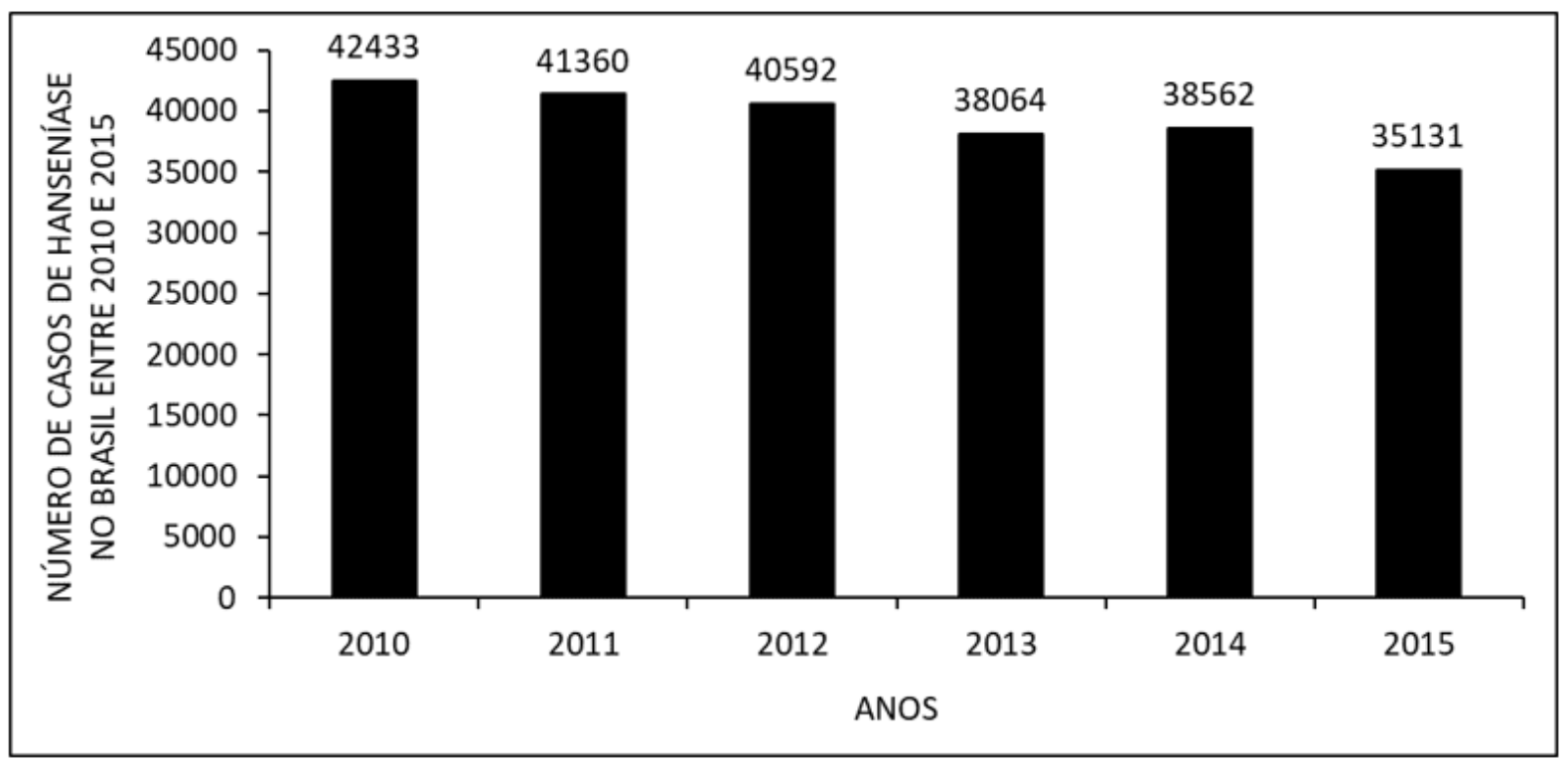

La Figura 2 muestra el número de casos de lepra en Brasil entre 2010 y 2015, por regiones del país. Los datos muestran que el mayor número de visitas ocurre en el noreste y la minoría en el sur.

RC: 68072

Disponible: https://www.nucleodoconhecimento.com.br/salud/hanseniase-in-brasil 
La Figura 2 muestra el número de casos de lepra en Brasil entre 2010 y 2015, por regiones del país.

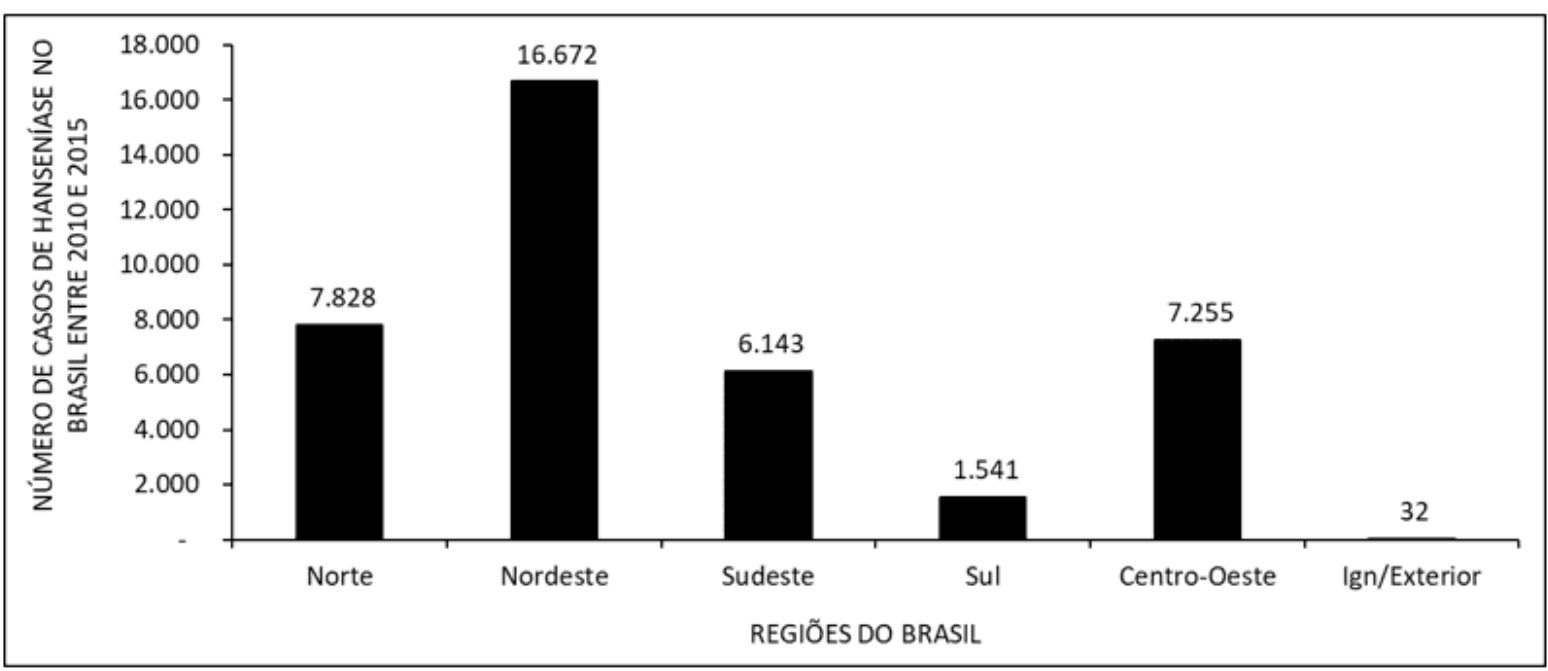

La Figura 3 muestra el número de casos de lepra en Brasil entre 2010 y 2015, según el género. Donde se observa que hay un mayor número de ocurrencias en los machos en comparación con las hembras.

RC: 68072

Disponible: https://www.nucleodoconhecimento.com.br/salud/hanseniase-in-brasil 
La Figura 3 muestra el número de casos de lepra en Brasil entre 2010 y 2015, según el género.

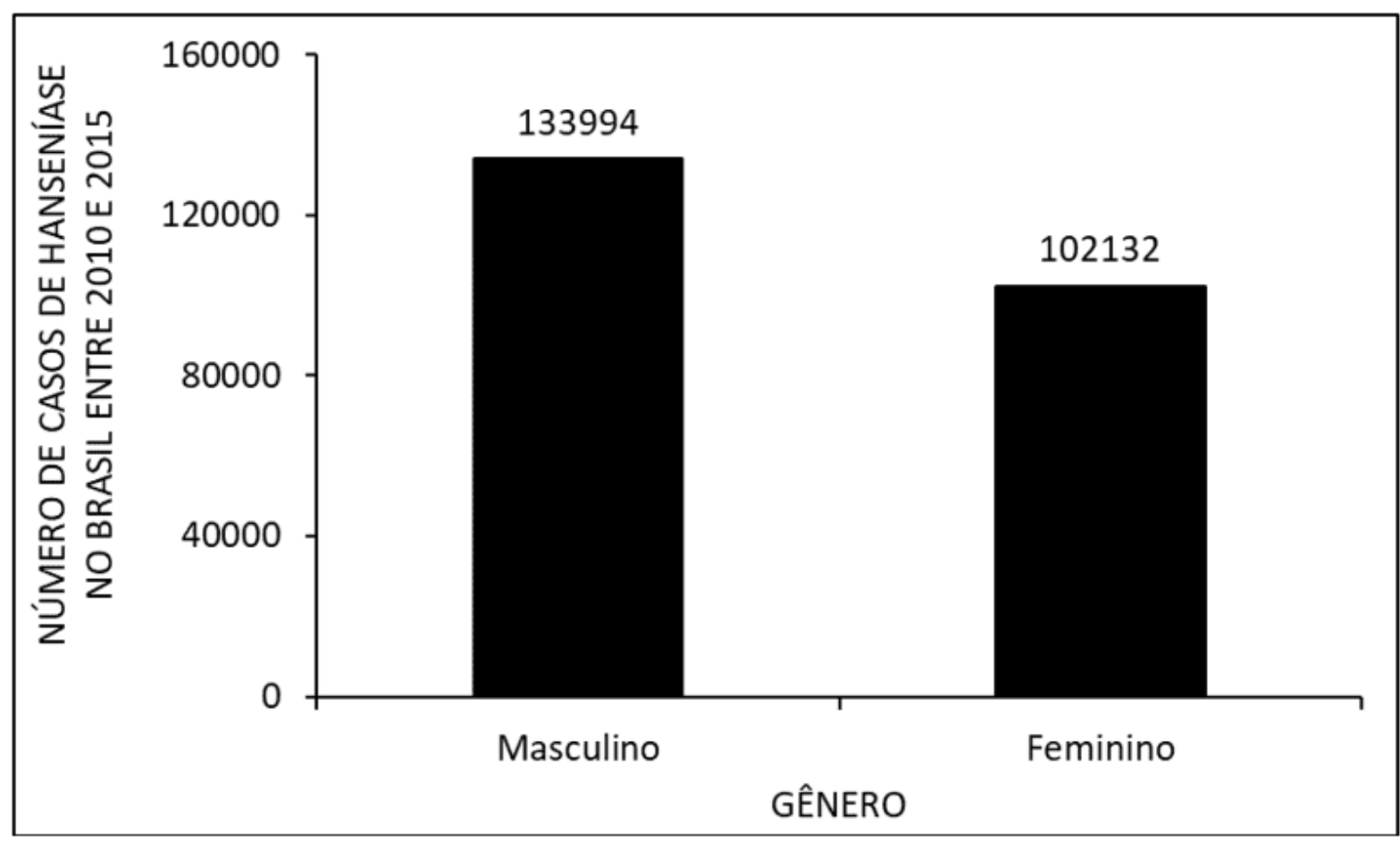

La Figura 4 muestra el número de casos de lepra en Brasil entre 2010 y 2015, por grupo de edad. Cuando se indique una mayor tasa de aparición de la enfermedad en adultos de 20 a 64 años, y menor en niños de 1 a 9 años y ancianos de 80 años o más.

RC: 68072

Disponible: https://www.nucleodoconhecimento.com.br/salud/hanseniase-in-brasil 
La Figura 4 muestra el número de casos de lepra en Brasil entre 2010 y 2015, por grupo de edad.

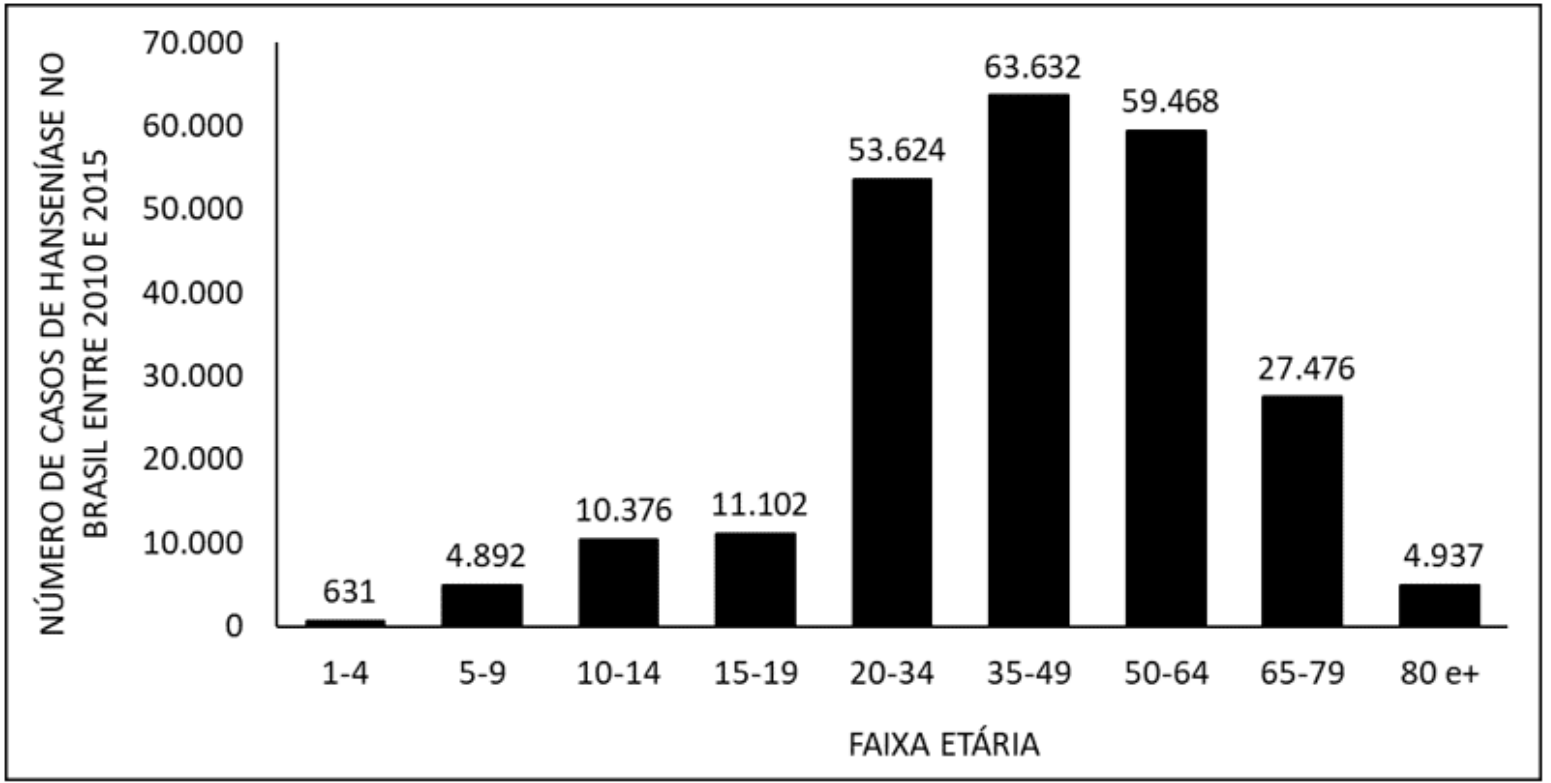

La Figura 5 muestra el número de casos de lepra en Brasil entre 2010 y 2015, según el modo de detección. El principal modo de detección de la enfermedad son las derivaciones, seguidas de la demanda espontánea.

RC: 68072

Disponible: https://www.nucleodoconhecimento.com.br/salud/hanseniase-in-brasil 
Figura 5 Muestra el número de casos de lepra en Brasil entre 2010 y 2015, según el modo de detección.

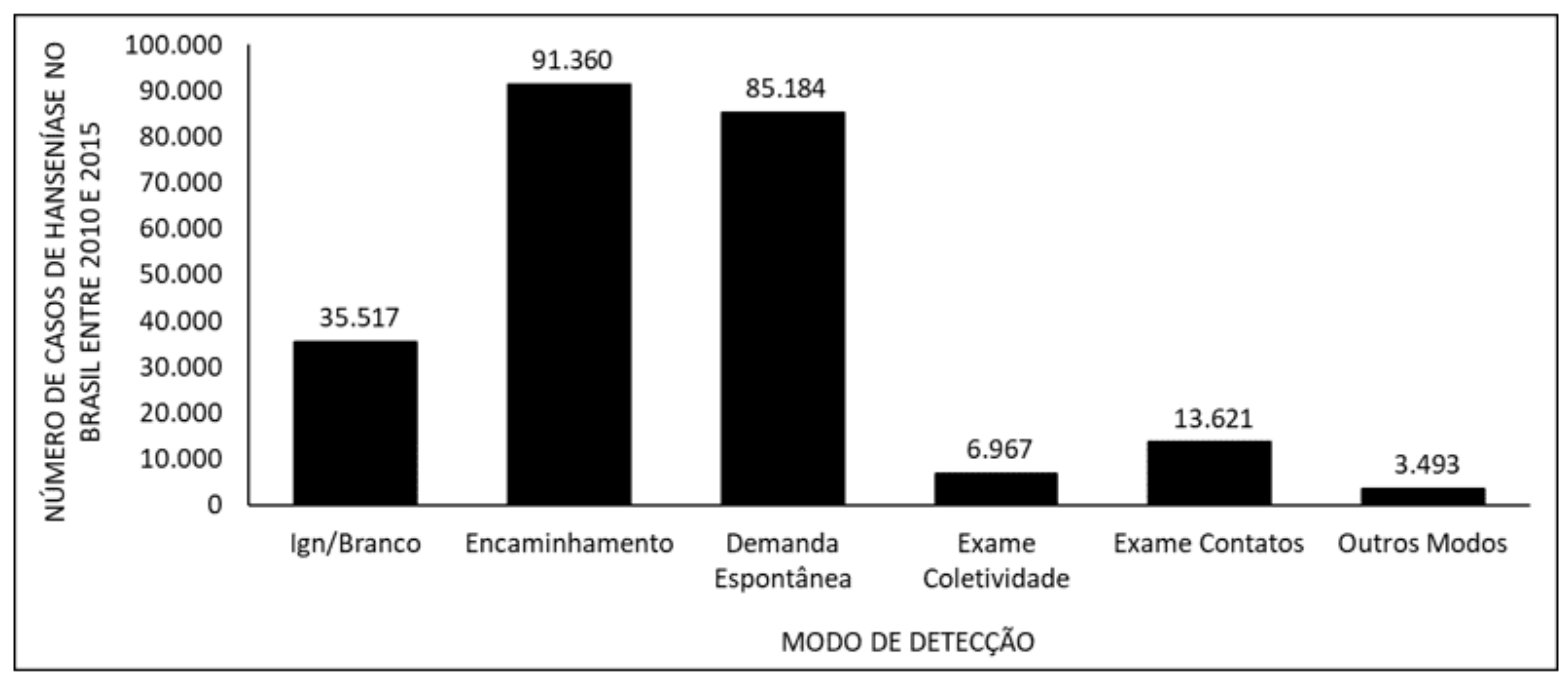

La Figura 6 muestra el número de casos de lepra en Brasil entre 2010 y 2015, debido a tipos de lesión cutánea. Los datos muestran que la mayoría de los pacientes tienen de 2 a 5 lesiones cutáneas y la minoría no presenta ninguna lesión.

RC: 68072

Disponible: https://www.nucleodoconhecimento.com.br/salud/hanseniase-in-brasil 
La Figura 6 muestra el número de casos de lepra en Brasil entre 2010 y 2015, por tipo de lesión cutánea.

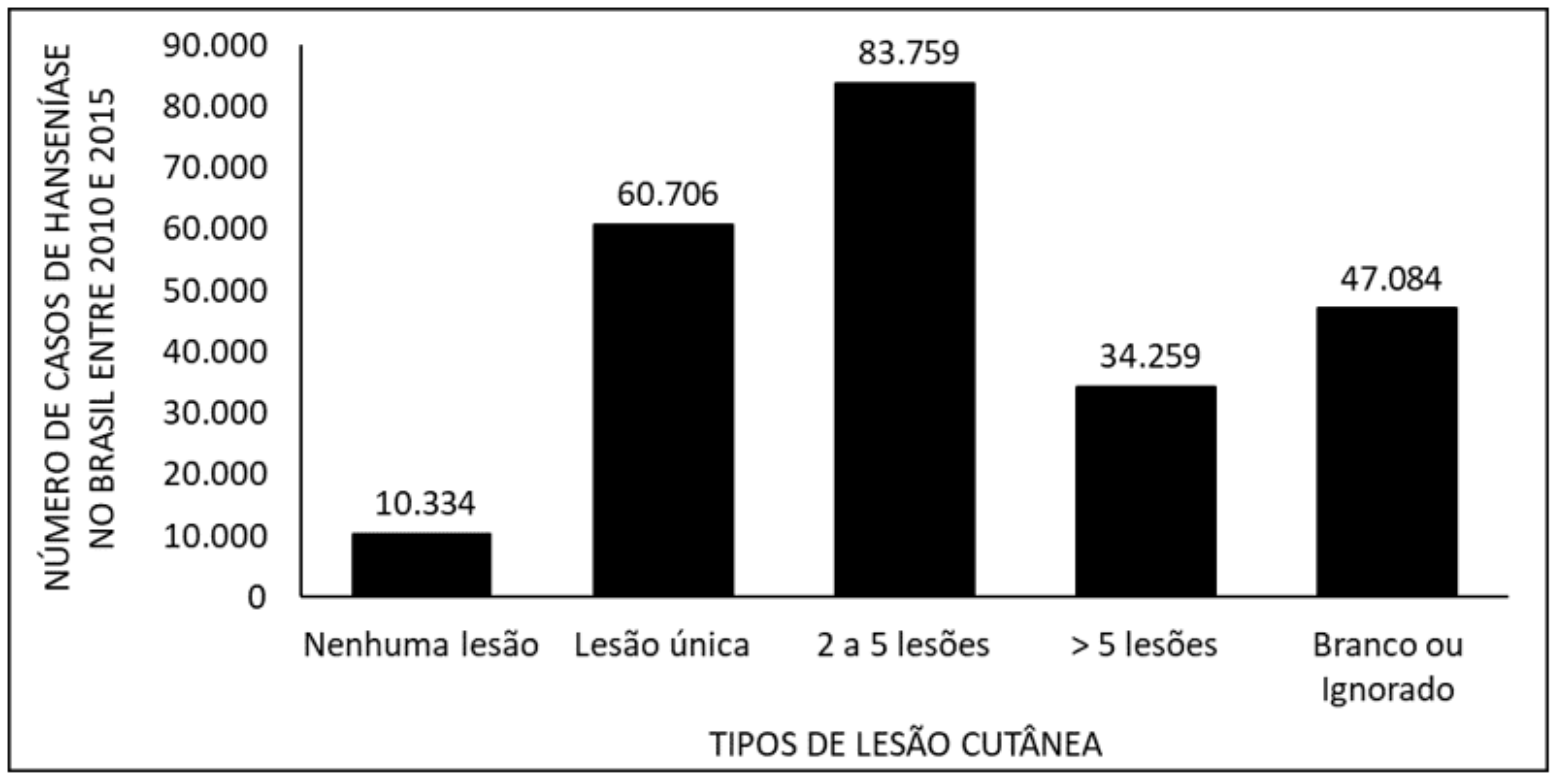

La Figura 7 muestra el número de casos de lepra en Brasil entre 2010 y 2015, mediante una evaluación diagnóstica. Los datos muestran que la mayoría de los casos pertenecen al grado cero y a la minoría del grado II de la enfermedad.

RC: 68072

Disponible: https://www.nucleodoconhecimento.com.br/salud/hanseniase-in-brasil 
La Figura 7 muestra el número de casos de lepra en Brasil entre 2010 y 2015, mediante una evaluación diagnóstica.

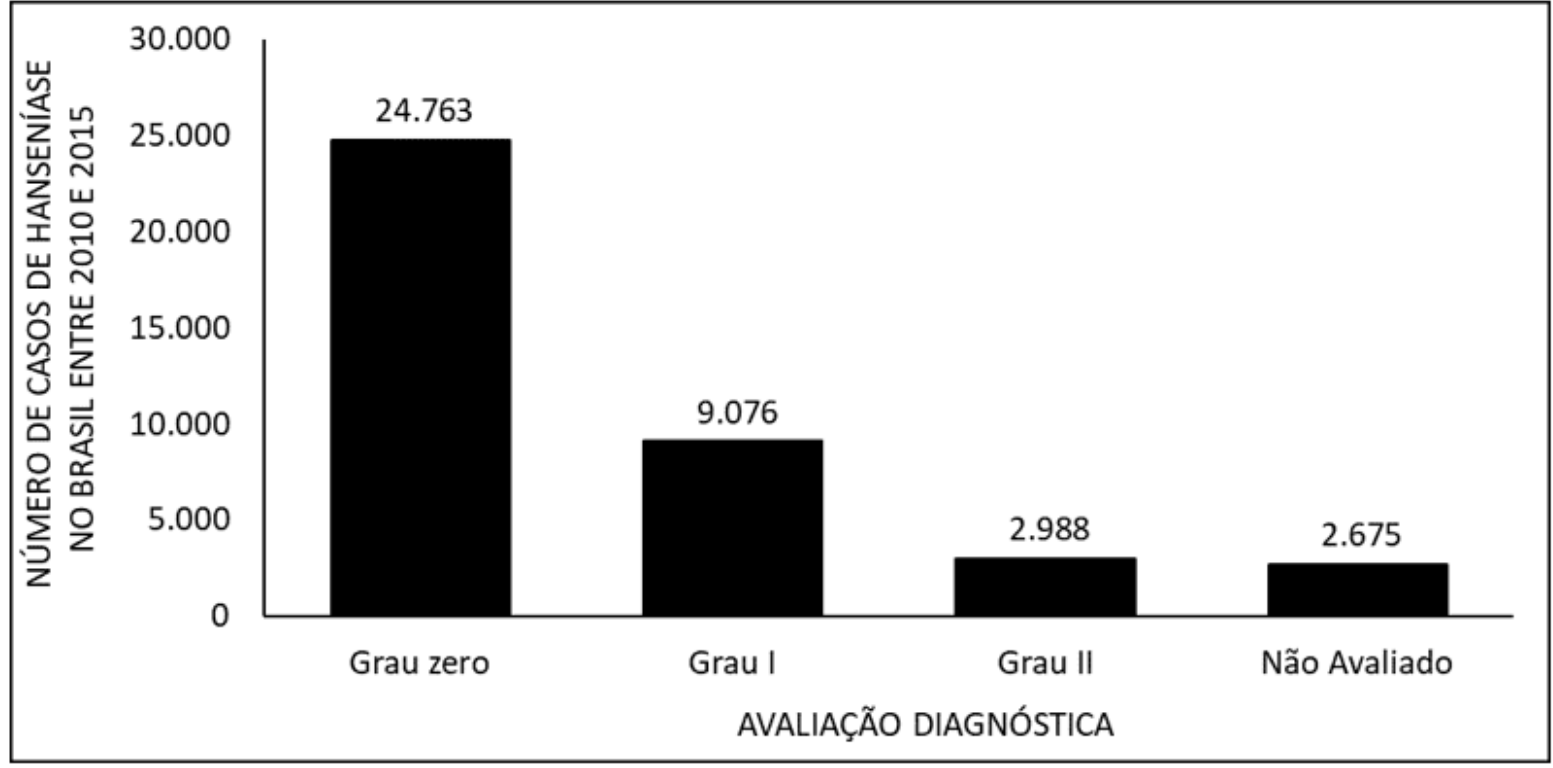

\section{DISCUSIÓN}

Durante los años 2010 a 2015, hubo una disminución en el número de pacientes con lepra, con un pequeño aumento en 2014 (figura 1). Los datos son corroborados por la literatura. La disminución probablemente se produjo a través de la implementación de políticas públicas para hacer frente a la enfermedad, como el diagnóstico precoz y el tratamiento exitoso, lo que conduce a la curación. El aumento en el número de 2014 se debe probablemente a la campaña para detectar nuevos casos de la enfermedad (BRASIL, 2015; 2017; 2017a).

El número de casos de lepra muestra más visitas en el noreste y menos en la región sur (figura 2). Según la literatura, este alto número de personas infectadas en el noreste se debe probablemente a la alta tasa de transmisión, que ocurre en las temporadas festivas. En un momento en que las unidades básicas de salud no actúan. En la región sur, la causa probable del bajo índice de pacientes se refiere a la distancia entre los servicios de salud y el paciente, obstaculizando el acceso y

$\mathrm{RC}: 68072$

Disponible: https://www.nucleodoconhecimento.com.br/salud/hanseniase-in-brasil 
contribuyendo a la evolución de la enfermedad, a un alto grado de discapacidad física (ROCHA, 2015).

Según el género, durante los años 2010 a 2015, los hombres tuvieron una mayor incidencia de la enfermedad que las mujeres (figura 3). Al comparar los datos con la literatura, se observa que la causa probable de esta alta tasa se debe al hecho de que los hombres están más expuestos a la enfermedad que a las mujeres, tomando tiempo para buscar servicios de salud y abandonar el tratamiento antes de la cura. Esto lo hace susceptible a desarrollar la deformidad característica de la enfermedad (ARAGOSO et al., 2014; ARANTES et al., 2010).

A nivel de ocurrencia, predominan los individuos de grupo de edad superior entre los infectados. Uno de los factores predominantes para este aspecto es el tiempo de incubación de la bacteria (Mycobacterium leprae), que puede extenderse de dos a siete años y, por lo tanto, presentándose en la edad adulta y, junto con esto, termina culminando en posibles implicaciones psicológicas, ya que, con la aparición de problemas físicos - amplificados por el retraso del diagnóstico - el paciente puede encontrarse incapaz de realizar su actividad profesional y su actividad profesional y , por lo tanto, dificultad para contribuir económicamente en el entorno familiar. Además, con el caso de casos familiares, se puede aumentar la probabilidad de enfermedad de los niños (VELÔSO, 2018).

Para el diagnóstico de la lepra, y su consecuente clasificación y puesta en escena, se analizan varios aspectos. Estos factores pueden variar según la clasificación utilizada, dos de los cuales son los principales, Ridley y Jopling's, basados en características histopatológicas, clínicas, bacilloscópicas e inmunológicas; la Clasificación de la Organización Mundial de la Salud (OMS) emplea el índice bacilloscópico en su clasificación, estableciendo que, si hay un máximo de cinco lesiones a nivel cutáneo y tronco nervioso, la lepra paucibacilar es. En caso de que haya más de cinco lesiones, a nivel cutáneo y tronco nervioso, hay lepra multibacilar. Sin embargo, si la microscopía de frotis de esputo está disponible en el paciente y se

RC: 68072

Disponible: https://www.nucleodoconhecimento.com.br/salud/hanseniase-in-brasil 
cumple el resultado positivo, la clasificación se basa automáticamente en multibacillar (LASTÓRIA y ABREU, 2012).

Dependiendo de la forma de manifestación de la lepra, varias afectaciones pueden estar físicamente presentes en el individuo infectado cuyo bacilo está activo, entre ellas: lesiones granulomatosas (que pueden surgir en diferentes formas, como nódulos o pápulas), edema, síntomas causados cuando los troncos nerviosos se ven afectados (como atrofia muscular, debilidad y dolor), problemas inmunológicos, sensoriales, debilidades motoras, procesos inflamatorios agudos y sistémicos y otras implicaciones para el cuerpo (PESSOA , 2019).

La lepra, con el fin de no desencadenar una afectación grave para el individuo, debe diagnosticarse a tiempo, utilizando la lógica clínica y las pruebas recomendadas. Si el diagnóstico ocurre tarde, las implicaciones pueden ser mayores. Además, la epidemiología y su control son necesarios para un amplio conocimiento de la situación de prevalencia de la enfermedad, reflejando así en el número de casos, ya que, si el control no es eficaz, puede haber una mayor difusión de la enfermedad y, en consecuencia, un aumento significativo en el número de casos. La búsqueda de ayuda médica en la etapa inicial de la enfermedad culmina en la prevalencia de casos pertenecientes al grado cero (en los que se preservan los segmentos musculares y sus respectivas sensibilidades, caracterizando, en gran parte, un buen pronóstico del paciente) (PESSOA, 2019; BRASIL, 2018).

\section{CONCLUSIÓN}

Con el avance de las políticas públicas encaminadas a combatir la lepra, basadas en el diagnóstico y tratamiento precoz, el número de casos de la enfermedad en Brasil, en el nivel mayoritario, disminuyó entre los años 2010 y 2015.

La lepra tiene una mayor prevalencia en los varones, y la causa probable de esta realidad es la mayor exposición de los hombres a esta patología, ya que la

$\mathrm{RC}: 68072$

Disponible: https://www.nucleodoconhecimento.com.br/salud/hanseniase-in-brasil 
búsqueda del servicio sanitario es menos global y, además, el mayor abandono del tratamiento, lo que resulta en el avance de la enfermedad y la consiguiente aparición de debilidades orgánicas del individuo.

Además, la tasa más alta de pacientes se encuentra en grupos de edad más grandes, es decir, en adultos, y el factor principal es responsable de ello el período de incubación de la enfermedad (que puede variar de 2 a 7 años). En vista de esto, se pueden desencadenar varios problemas a nivel individual, como las implicaciones físicas debilitantes y psicológicas, y la familia, porque, con la aparición de la debilidad, pueden surgir dificultades económicas debido a la pérdida de la actividad profesional remunerada.

Con el fin de reducir el número de casos de la enfermedad en el país y poder hacer el diagnóstico lo antes posible, se deben analizar los hallazgos clínicos y las pruebas disponibles para esta detección. Si el paciente puede hacerse su diagnóstico en la etapa temprana de la enfermedad, por lo general hay un buen pronóstico, y su salud orgánica se puede preservar en gran medida, manteniendo la sensibilidad e integridad de sus segmentos musculares.

\section{REFERENCIAS}

ARAGOSO, I.; CARVALHO, R.M.B.; SOUSA, C.M.; VIEIRA, G.D. Hanseníase em Rondônia: incidência e características dos casos notificados, 2001 a 2012. Epidemiologia e Serviços de Saúde, v.23, n.2, Brasília, jun. 2014.

ARANTES, C.K.; FILIPE, M.S.; GARCIA, M.L.R.; NARDI, M.T.; PASCHOAL, V.D.A. Avaliação dos serviços de saúde em relação ao diagnóstico precoce da hanseníase. Epidemiologia e Serviços de Saúde, v.19, n.2, Brasília, jun. 2010.

BRASIL. Ministério da Saúde. Secretaria de Políticas de Saúde. Departamento de Atenção Básica. Guia para o controle da hanseníase. $1^{\underline{a}}$ ed., Brasília (DF): Ministério

RC: 68072

Disponible: https://www.nucleodoconhecimento.com.br/salud/hanseniase-in-brasil 
da

Saúde,

2002.

Disponível

em:

<http://bvsms.saude.gov.br/bvs/publicacoes/guia_de_hanseniase.pdf> Acesso em: 24 ago 2017.

BRASIL. Ministério da Saúde. Secretaria de Vigilância em Saúde. Departamento de Vigilância das Doenças Transmissíveis. Diretrizes para vigilância, atenção e eliminação da Hanseníase como problema de saúde pública: manual técnicooperacional. 1 $1^{\underline{a}}$ ed., Brasília (DF): Ministério da Saúde, 2016. Disponível em: <http://www.saude.pr.gov.br/arquivos/File/DiretrizesdoManuaTcnicoOperacionaldeH ansenase.pdf> Acesso em: 05 set 2017.

BRASIL. Ministério da Saúde. Secretaria de Vigilância em Saúde. Departamento de Vigilância das Doenças Transmissíveis. Eliminar a hanseníase é possível: um guia para os municípios. Brasília (DF): Ministério da Saúde, 2015. Disponível em: <http://bvsms.saude.gov.br/bvs/publicacoes/eliminar_hanseniase_possivel_versao_p reliminar.pdf> Acesso em: 05 set 2017.

BRASIL. Boletim Epidemiológico. Brasilía DF. Ministério da Saúde. 49, n. 4, 12p. 2018.

FREITAS, A.A. Resposta imune celular e humoral a proteínas recombinantes do Mycobacterium leprae em pacientes com outras dermatoses. Tese (Doutorado) Universidade Federal de Goiás, Instituto de Patologia Tropical e Saúde Pública, Program de Pós Graduação em Medicina Tropical e Saúde Pública, Goiânia, 2015. Disponível em: $<$ https:// posstrictosensu.iptsp.ufg.br/up/59/o/AlinedeAraujoFreitas_2015_vers\%C3\%A3ofinal. pdf> Acesso em: 12 set 2017.

LASTÓRIA, J. C; ABREU, M. A. M. M. Hanseníase: diagnóstico e tratamento. Diagn Tratamento, v. 17, n. 4, p. 173-179, 2012.

$\mathrm{RC}: 68072$

Disponible: https://www.nucleodoconhecimento.com.br/salud/hanseniase-in-brasil 
OLIVEIRA, Ciane Martins de; et. al. A evolução da assistência ao paciente com Hanseníase: dos leprosários à poliquimioterapia. Revista Científica Multidisciplinar Núcleo do Conhecimento. Ano 01, Vol. 06, Ed. 08, pp. 68-80. Agosto de 2016. ISSN: 2448-0959

PESSOA, M. M. S. F. S. Hanseníase no Brasil: Uma revisão literária, nos anos de 2014 a 2019. 45p. Trabalho de Conclusão de Curso (Graduação de Farmácia) Universidade Federal do Rio Grande do Norte, Rio Grande do Norte, Natal, 2019.

ROCHA, A.C.A.A. Análise da sazonalidade da incidência de hanseníase segundo regiões geográficas, climas e biomas do Brasil. 2015. 82 f. Dissertação (Mestrado em Ciências Ambientais) - Universidade do Estado de Mato Grosso, Programa de Pós-Graduação em Ciências Ambientais, Cáceres/MT.

VELÔSO, D. S. Perfil clínico-epidemiológico da hanseníase no estado do Piauí, no período de 2009 a 2016. 127p. Dissertação (Mestrado em Medicina Tropical) Instituto Oswaldo Cruz, Piauí, Teresina, 2018.

Artículo: Noviembre de 2020.

Aprobado: Noviembre, 2020.

RC: 68072

Disponible: https://www.nucleodoconhecimento.com.br/salud/hanseniase-in-brasil 